and most frequently in the elderly pts with arterial hypertension. However, the fact of the correlation between diastolic disfunction and high level of ESR $(r=0,364, \mathrm{p}<0,05)$ as well as fibrinogen concentration $(\mathrm{r}=0,325, \mathrm{p}<0,05)$ and reduction of capillary loops do not exclude the influence of sclerodermic fibrosis on these abnormalities. Mitral (22\%) and aortal (5\%) regurgitations were the most frequent indication of endocard damage, however, there was no significant difference between these abnormalities and other clinical manifestations of SSc. Fifty one out of 100 pts (51\%) were diagnosed as having pulmonary hypertension $(\mathrm{PH})$, including 15 pts having isolated $\mathrm{PH}$ without other antecedent pulmonary or cardiac conditions. Correlations were found between PH and age of pts, high level of ESR and fibrinogen concentration. Twenty-six pts out of 100 did not complain on cardiac problem, but Doppler Ehocardiography studies showed pericarditis in two cases, diastolic abnormality in three cases, mitral regurgitation in four cases and pulmonary hypertension also in four cases.

Conclusion Doppler Ehocardiography is an important method in diagnosis of cardial involvement in SSc pts including those without any symptoms of sclerodermic cardiac disease.

\section{FRI0211 SERUM CARNITINE MEASUREMENT IN PRIMARY RAYNAUD'S PHENOMENON}

${ }^{1} \mathrm{~L}$ La Civita, ${ }^{2} \mathrm{G}$ Pugliese, ${ }^{1} \mathrm{P}$ Fadda, ${ }^{1} \mathrm{O}$ Olivieri. ${ }^{1}$ Rheumatology Department of Lucania; ${ }^{2}$ Lab Unit, San Carlo Hospital, Potenza, Italy

\subsection{6/annrheumdis-2001.292}

Background Raynaud's phenomenon (RP) is a microcirculatory disorder characterised by deficient endothelial activity. Such endothelial dysfunction should be responsible for reduced release of NO, a potent vasodilatory agent. Since the cell function is dependent on energy production, it is though deficient endothelial activity could be due to the loss of energy production probably secondary to a low availability of Carnitine, an important cell metabolic carrier.

Objectives To conduct a preliminary evaluation of the serum Carnitine concentrations in primary RP patients (pRP pts.) compared to age- and sex-matched controls.

Methods After verbal consent, 17 consecutive pRP pts (16 female, mean age $32.1 \pm 11.3 \mathrm{yrs}$ ) and ten control subjects (9 female, mean age $34 \pm 11$ yrs) were enrolled for this study. Patients had to have a RP history no longer than 5 years. After acclimatisation, each subject underwent a venous blood drawing from same vessel at baseline and after a 5-minute cold-water test. All blood samples were stored $? 20^{\circ} \mathrm{C}$. Then, Carnitine was measured in collected sera by enzymatic assay (SIGMA-TAU pharmaceuticals). T-test for paired data was used for statistical analysis.

Results Table 1 showed the mean values of serum Carnitine concentrations in two studied groups. After test, serum Carnitine concentration increased in $12 / 17 \mathrm{pRP}$ patients and lowered in the remainders. Contrary, in the control group circulating Carnitine increased significantly after provocative test.

Conclusion Lower circulating Carnitine titres in our pRP pts could be associated with diminished availability of Carnitine in endothelial cells and consequently with their impaired activity. Although this study is preliminary, our results suggest Carnitine supplementation for promotion of endothelial activity in pRP pts may be worth investigating, particularly in RP subjects unresponsive to conventional treatments.

\begin{tabular}{llll}
\multicolumn{4}{l}{ Abstract FRI0211 Table 1} \\
\hline & Baseline & After test & p-value \\
\hline pRP (17, mg/L) & $34.4 \pm 16.7 \pm 1.9$ & $35.9 \pm 6.9 \pm 1.7$ & n.s. \\
Controls (10, mg/L) & $33.5 \pm 16.1 \pm 5.1$ & $42.7 \pm 19.5 \pm 6.2$ & 0.0005 \\
\hline
\end{tabular}

\section{OP0036 MOLECULAR CLONING AND CHARACTERISATION OF AUTOANTIGEN RECOGNISED BY ANTI-WA ANTIBODIES IN PATIENTS WITH SYSTEMIC SCLEROSIS}

${ }^{1} Y$ Shirai, ${ }^{1} \mathrm{~T}$ Nojima, ${ }^{1} \mathrm{M}$ Hirakata, ${ }^{2} \mathrm{H}$ Yamagata, ${ }^{3} \mathrm{~K}$ Miyachi, ${ }^{4} \mathrm{~T}$ Mimori. ${ }^{1}$ Internal Medicine, Keio University; ${ }^{2}$ Internal Medicine, Natl. Murayama. Hospital, Tokyo; ${ }^{3}$ Internal Medicine, Keigu Clinic, Yokohama; ${ }^{4}$ Clinical Immunology, Kyoto University, Kyoto, Japan

\subsection{6/annrheumdis-2001.293}

Background Anti-Wa antibodies were first reported in patients with systemic sclerosis (SSc) as autoantibodies reactive with a $48 \mathrm{kDa}$ transfer RNA-associated protein. ${ }^{1}$ However, the nature of the $\mathrm{Wa}$ antigen has not been clarified so far.

Objectives To identify and characterise the Wa antigen by molecular cloning using anti-Wa serum as a probe.

Methods Molecular cloning of the target Wa antigen was performed using lambda gt11 cDNA library constructed from human liver mRNA and anti-Wa protoype serum as a probe. Bound antibodies were eluted from the fusion proteins of the isolated clones expressed in E.coli and were used for RNAimmunoprecipitation assay. The reactivity of fusion proteins prepared from lysogenic E.coli Y1089 carrying the recombinant phages were examined using five sera that were proved to have anti-Wa antibodies in double immunodiffusion assay. The cDNA inserts of the isolated clones were sequenced, and the detailed homologies of nucleotide and amino acid sequences were analysed through the BLAST server at NCBI DNA databank. AntiWa antibodies in sera from patients with various rheumatic diseases were detected by immunoblot analysis using the fusion protein from the positive clone.

Results 10 positive clones were recognised by the probe serum. In RNA-immunoprecipitation, the affinity-purified antibodies from only one clone (termed Wa-1) immunoprecipitated transfer RNA from HeLa cell extracts. In immunoblot study, the Wa-1 fusion protein was recognised by all five sera that were proved to have anti-Wa antibodies. These data confirmed that the Wa-1 clone encoded for the Wa antigen. The nucleotide sequence and deduced amino acid sequence of the $0.7-\mathrm{kb}$ insert cDNA of Wa1 were completely identical with the C-terminal part of NEFA (named after DNA-binding domain, two EF-hands, Acidic amino acid rich region)/nucleobindin-2. ${ }^{2}$ In immunoblots using the Wa1 fusion protein, autoantibodies to Wa (NEFA/nucleobindin-2) were detected in 3 of 21 SSc (21\%), one of 10 RA (10\%) and one of 16 SLE sera (6\%). However, neither RA nor SLE sera immunoprecipitated transfer RNA from HeLa cells, while all 3 SSc sera immunopricipitated transfer RNA, suggesting the diversity of autoepitopes among patient sera.

Conclusion These results demonstrated that the autoantigen recognised by anti-Wa antibodies were proved to be identical with NEFA/nucleobindin-2.

\section{REFERENCES}

1 Miyachi K, et al. J Rheumatol. 1991;18:373-8

2 Barnikol-Watanabe S, et al. Biol Chem Hoppe Seyler 1994;375:497-512 